ARTICLE

Received 1 Nov 2012 | Accepted 5 Apr 2013 | Published 7 May 2013

DOl: $10.1038 /$ ncomms2837

OPEN

\title{
Select interneuron clusters determine female sexual receptivity in Drosophila
}

Akira Sakurai ${ }^{1 \dagger \dagger}$, Masayuki Koganezawa ${ }^{1}$, Kei-ichiro Yasunaga $^{2}$, Kazuo Emoto $^{2}$ \& Daisuke Yamamoto ${ }^{1}$

Female Drosophila with the spinster mutation repel courting males and rarely mate. Here we show that the non-copulating phenotype can be recapitulated by the elimination of spinster functions from either spin-A or spin-D neuronal clusters, in the otherwise wild-type (spinster heterozygous) female brain. Spin-D corresponds to the olfactory projection neurons with dendrites in the antennal lobe VA1v glomerulus that is fruitless-positive, sexually dimorphic and responsive to fly odour. Spin-A is a novel local neuron cluster in the suboesophageal ganglion, which is known to process contact chemical pheromone information and copulation-related signals. A slight reduction in spinster expression to a level with a minimal effect is sufficient to shut off female sexual receptivity if the dominant-negative mechanistic target of rapamycin is simultaneously expressed, although the latter manipulation alone has only a marginal effect. We propose that spin-mediated mechanistic target of rapamycin signal transduction in these neurons is essential for females to accept the courting male.

\footnotetext{
${ }^{1}$ Division of Neurogenetics, Tohoku University Graduate School of Life Sciences, Sendai 980-8577, Japan. ${ }^{2}$ Department of Cell Biology, Osaka Bioscience Institute, Suita 565-0874, Japan. 'Present address: Department of Neurobiology, University of Massachusetts Medical School, LRB 705, 364 Plantation Street, Worcester, Massachusetts 01605-4321, USA. Correspondence and requests for materials should be addressed to D.Y. (email: daichan@m.tohoku.ac.jp).
} 
$\mathrm{F}$ emale partner preference is believed to be a major driving force for the creation of male-specific morphology and behaviour that improve the reproductive success of males ${ }^{1}$. One of the factors modulating female partner preference is sexual receptivity, the physiological mechanism of which has been poorly understood. Except for a few days after eclosion, wild-type virgin females in Drosophila melanogaster copulate when courted by a male ${ }^{2}$. In contrast, females with the spinster (spin) mutation resist copulating. The spin mutant females display a variety of rejection behaviours against a courting male, such as decamping, fending with the legs, flicking of the wings, kicking, curling the abdomen and spreading the vaginal plate ${ }^{3,4}$. In this study, we intend to identify the central nervous system neurons that control sexual receptivity and thus partner preference of female flies by producing a small number of spin mutant homozygous cells in the otherwise spin heterozygous brain, with the expectation that such spin-mosaic virgin females that have spin mutant clones in the 'receptivity centre' will repel a courting male in contrast to the remainder mosaic females with spin mutant clones in the brain regions unrelated to receptivity. The unambiguous behavioural phenotype is a prerequisite for this type of analysis and spin mutants suffice this requirement. To generate spin mutant clones we adopted mosaic analysis with a repressible cell marker (MARCM $)^{5}$, with which the decision-making centre for male courtship behaviour has been identified $^{6,7}$. Our analysis successfully identify two interneuron groups that induce non-copulating phenotype in females when they are homozygous for the spin mutation. We identify the spin-D group, which is composed of second-order olfactory projection neurons that relay the conspecific odour information, and the spin-A group, which represents a local neuron cluster confined in the suboesophageal ganglion. We propose that spin functions as a molecular switch in these neurons to convert the female physiology from the sexually non-receptive to receptive state and to coordinate the associated changes in mating behaviour.

\section{Results}

Neural and glial Spin coordinately regulates receptivity. The receptivity of spin mutant females is restored by spin ${ }^{+}$overexpression, as driven by spin-GAL4 with expression in both glia and neurons (Fig. 1a-c). To determine whether the expression in glia or neurons or both is crucial for the spin behavioural role, we attempted to rescue the mutant phenotype by selectively overexpressing spin $^{+}$in neurons with elav ${ }^{\text {c155 }}$ (referred to as elavGAL4 hereafter) or in glia with repo-GAL4. The results indicated that neuronal but not glial overexpression of $\operatorname{spin}^{+}$restores the receptivity that has been reduced by the spin mutation (Fig. 1d). In the second set of experiments, spin functions were knocked down in either neurons or glia by forcibly expressing UAS-spin $R N A i$ as driven by elav-GAL4 or repo-GAL4. The flies were raised at $29^{\circ} \mathrm{C}$ to enhance transgene expression and tested for mating behaviour at $25^{\circ} \mathrm{C}$. We found that the spin RNAi expressed in neurons but not that expressed in glia attenuated the sexual receptivity of wild-type females (Fig. 1e). To further evaluate the possible involvement of spin function in glia, spin was knocked down in both neurons and glia in flies raised at $25^{\circ} \mathrm{C}$, a temperature expected to result in low-level expression of transgenes. Interestingly, when spin RNAi was expressed in both neurons and glia, female sexual receptivity dramatically declined even in these female flies raised at $25^{\circ} \mathrm{C}$ (Fig. 1f), the temperature at which no effect was detected if spin RNAi was targeted to either neurons or glia. We conclude that female receptivity is established by spin functions primarily in neurons while glial spin has a subsidiary role.
Two neuronal clusters determine female sexual receptivity. To determine which neurons are involved in making the female flies sexually receptive, we conducted $\mathrm{MARCM}^{5}$ in which spin mutant clones were produced in the brain of spin heterozygous females, which were subjected to mating behaviour assays and subsequent histology to identify the neurons that were spin mutant clones (Fig. 2). Among the 902 mosaic females subjected to mating assays, 7 flies did not copulate in a 1-h observation period and the remaining 895 flies copulated. In our experimental design, only cells mutant for spin were labelled with mCD8::GFP. These cells showed strong lysotracker staining (Fig. 3), in keeping with the reported spin mutant phenotype ${ }^{8,9}$. Labelling the brains from these seven non-receptive females revealed several neuronal clusters that were mCD8::GFP positive in more than three flies, implying that some of these clusters are pivotal in regulating female receptivity; these included eight clusters that are reliably identifiable across different flies based on the location of their somata and neurite projections, and we named these clusters spin-A, $-\mathrm{B},-\mathrm{C},-\mathrm{D},-\mathrm{E},-\mathrm{F},-\mathrm{G}$ and $-\mathrm{H}$, respectively (Fig. 2a-f). To assess whether any of these clusters indeed contributes to the determination of sexual receptivity levels, we compared the frequency of homozygous (mCD8::GFP positive) cells in the respective clusters between the copulating and non-copulating mosaic fly groups. If any of these clusters have a strong impact on the sexual receptivity levels, then one can anticipate that such a cluster will be spin homozygous (mCD8::GFP positive) at a significantly higher frequency in the non-copulating fly group than in the copulating fly group. It turned out that the frequency of spin mutant homozygosity was significantly higher in the spinA and -D clusters of the non-copulating fly group than in those of the copulating fly group (Fig. $2 \mathrm{~g}$ ), but this relation was not observed for the other clusters. We have no evidence that some other clusters need to be spin mutant homozygous in addition to the spin-A and -D cluster in order to make the mosaic female unreceptive to a courting male (Table 1). We conclude that the spin-A and -D clusters participate in the regulation of female sexual receptivity.

Close inspection of the mCD8::GFP-labelled spin-D clones revealed that this cluster is composed of secondary projection neurons with dendritic fields in the antennal lobe (Fig. 2i) while extending their axons towards the lateral horn $(l h)$ via the mushroom body (Fig. 2j). Single clones of the spin-D cluster from different mosaic flies share six glomeruli that are positive for mCD8::GFP, that is, VA1d, VA1v, VA3, VM1, VM2 and VM5 (Fig. 2i). As every projection neuron is known to innervate a single glomerulus ${ }^{10}$, the spin-D cluster is considered to be a composite of six distinct types of projection neurons. On the other hand, the spin-A cluster represents a hitherto undescribed group of some 35-45 local neurons confined to the suboesophageal ganglion (Fig. 2h). Labelling of the spin-A cluster was inhibited by Choline-acetyltransferase-GAL80 (ChatGAL80) (Fig. 4a,b) and thus the neurons composing this cluster are likely to be cholinergic. Arbours of the spin-A cluster neurons interdigitate with axon terminals of $p p k$-positive neurons (Fig. $4 \mathrm{c}$ and Supplementary movie 1) whose origin remains obscure.

Or47b olfactory pathway regulates sexual receptivity. We wanted to determine whether all six types of projection neurons in the spin-D cluster are involved in female sexual receptivity, or only a few of these types are important. As no tool is available to manipulate each type of projection neurons separately, we instead attempted to prevent select projection neurons from receiving normal synaptic inputs by expressing a neural activity blocker or spin RNAi in primary afferents, for which distinct Or-GAL4s can be used to separately manipulate sensory inputs to different 
a

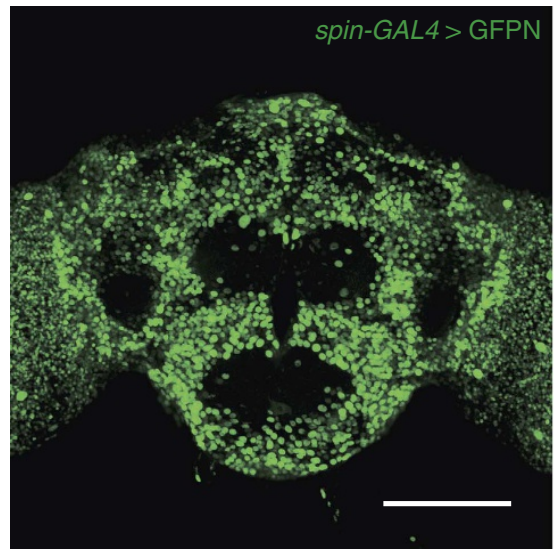

b

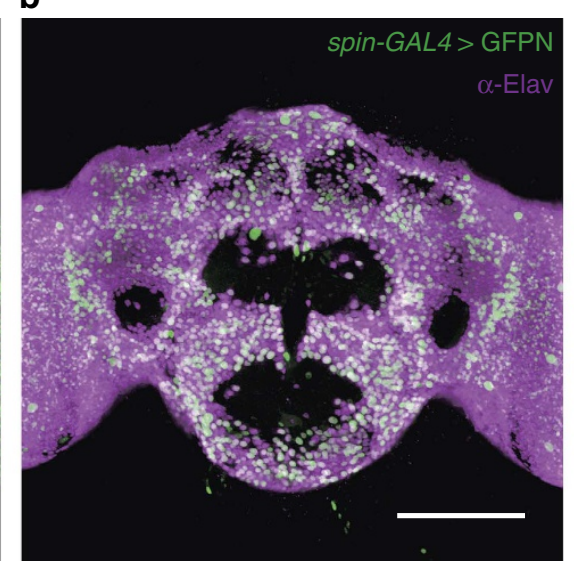

C

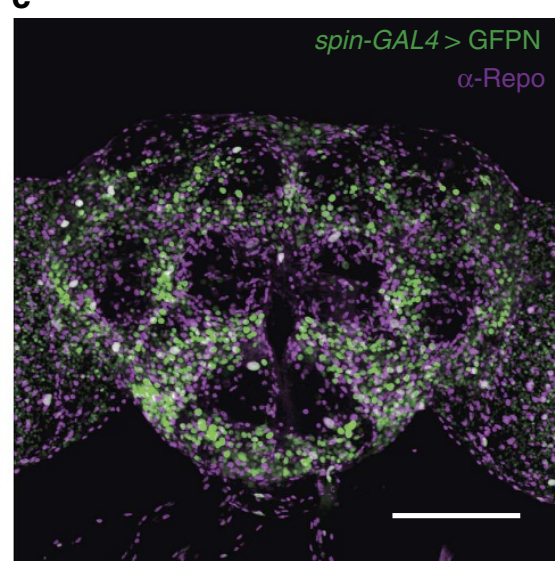

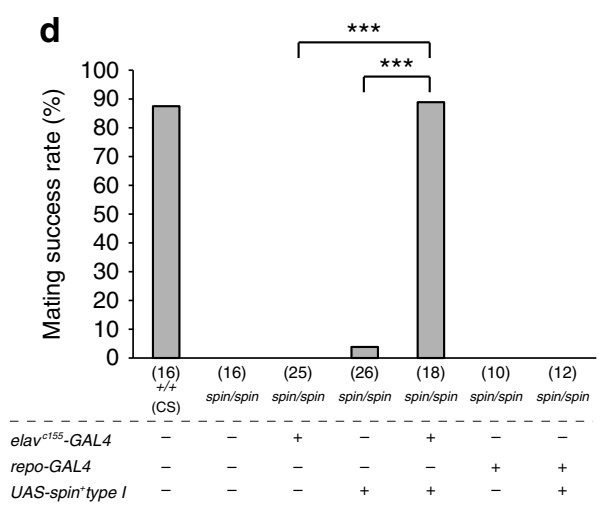

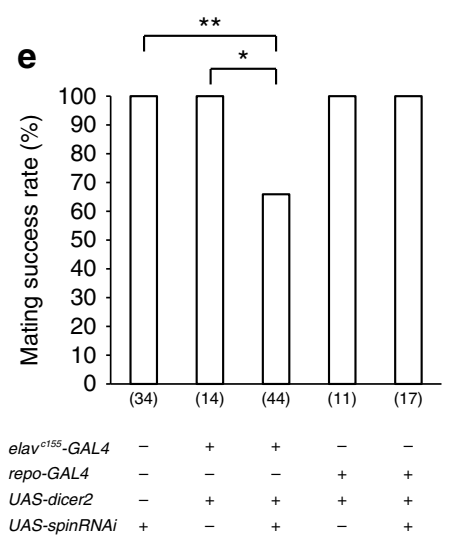

f

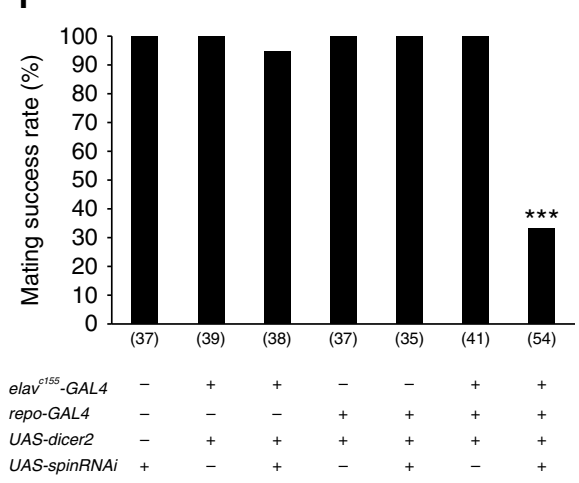

Figure 1 | Contributions of neuronal and glial Spin to female sexual receptivity. (a) spin-GAL4 expression in the adult female brain was detected with nuclear-targeted GFP (GFPN). (b,c) spin-GAL4-expressing cells are composed of anti-Elav-positive neurons (magenta in b) and anti-Repo-positive glia (magenta in $\mathbf{c}$ ) in the adult female brain. (d) The effectiveness of UAS-spin ${ }^{+}$type $/$in rescuing the non-copulating phenotype of spin ${ }^{P 1}$ mutant females was estimated by expressing it in neurons with elav ${ }^{c 155}$ or in glia with repo-GAL4. Wild-type (CS, Canton-S) and other control flies were also examined. The spin gene produces five isoforms, Spin I-V, only two of which (Spin I and Spin V) have been demonstrated to rescue the sexual receptivity phenotype ${ }^{4}$. Transgenes the fly carried are shown at the bottom; + and - indicate the presence and absence of the indicated transgene in each fly group. The number of flies examined is shown in parentheses. The female sexual receptivity was measured by the percentage of pairs copulating within a 1-h observation period at $25^{\circ} \mathrm{C}$. (e) The effect of spin knockdown in either neurons or glia on sexual receptivity when the flies were raised at $29^{\circ} \mathrm{C}$ to attain higher levels of transgene expression. Dicer2 was also used to enhance the RNAi effect. Neuronal but not glial expression of RNAi was effective in reducing sexual receptivity. (f) When the flies were raised at $25^{\circ} \mathrm{C}$, transgene expression levels would be lower than at $29^{\circ} \mathrm{C}$, spin RNAi expression in either neurons or glia had no discernible effect on receptivity, while its simultaneous expression in both neurons and glia reduced sexual receptivity. The statistical significance of differences was evaluated by the Fisher's exact probability test with Bonferroni correction $\left({ }^{\star \star \star} P<0.001 ;{ }^{\star \star} P<0.01 ;{ }^{\star} P<0.05\right)$. Simultaneous spin knockdown in neurons and glia was not possible at $29^{\circ} \mathrm{C}$ because practically no adult flies were recovered for lethality. Scale bar, $100 \mu \mathrm{m}$ for a-c.

glomeruli. The six glomeruli involving the spin-D cluster have been known to receive inputs from the following sensory neurons, each of which expresses a specific Ors ${ }^{11,12}$ : Or88a (VA1d), Or47b (VA1v), Or67b (VA3), unidentified (VM1), Or43b (VM2) and Or98a (VM5). We compared the effect of forced expression of an activated form of the inward rectifier $\mathrm{K}$ channel Kir2.1 (ref. 13), which hyperpolarizes the membrane and inhibits neural excitation, on female sexual receptivity when driven by five different Or-GAL4s, that is, Or88a-GAL4 (VA1d), Or47b-GAL4 (VA1v), Or67b-GAL4 (VA3), Or43b-GAL4 (VM2) and Or98aGAL4 (VM5). The results showed that Or98a- or Or47b-neurons, when inhibited, significantly retarded the copulation initiation, whereas Or43b-, Or67b- and Or88a-neurons did not (Fig. 5). The negative effect on the copulation success of inhibiting Or47b neurons was more pronounced compared with that of Or98a neurons (Fig. 5b versus 5e). Our preliminary findings show that rather unexpectedly, the reduced female receptivity caused by the inhibition of Or47b neurons was not further reduced by the additional inhibition of Or98a neurons (data not shown).
We next examined the effect of knockdown of spin in Or98a-, Or47b-, Or43b-, Or67b- or Or88a-neurons on female sexual receptivity. In this series of experiments, repo-GAL4 was combined with each Or-GAL4 to drive spin RNAi expression, because simultaneous knockdown of spin in neurons and glia was most effective in reducing female sexual receptivity (Fig. 6a-e). As can be seen in Fig. 6a-e, Or47b neurons, but none of the other four olfactory receptor neurons, attenuated female sexual receptivity when spin knockdown was achieved (Fig. 6a-e). We conclude that the olfactory pathway mediated by the Or $47 \mathrm{~b}$ neurons and the projection neurons with dendrites in the VA1v glomerulus has a key role in determining the level of female sexual receptivity. Among the five Ors examined, Or47b and Or88a are the receptors known to respond to conspecific fly odour (other Ors are activated by food odour, for example) ${ }^{14}$, and VA1v is one of the three sexually dimorphic glomeruli ${ }^{15,16}$ and expresses fruitless $(f r u)^{16}$, a major control gene for the courtship circuitry ${ }^{17,18}$. Notably, spin knockdown in Or47b neurons reduced but did not block female sexual receptivity, in contrast 

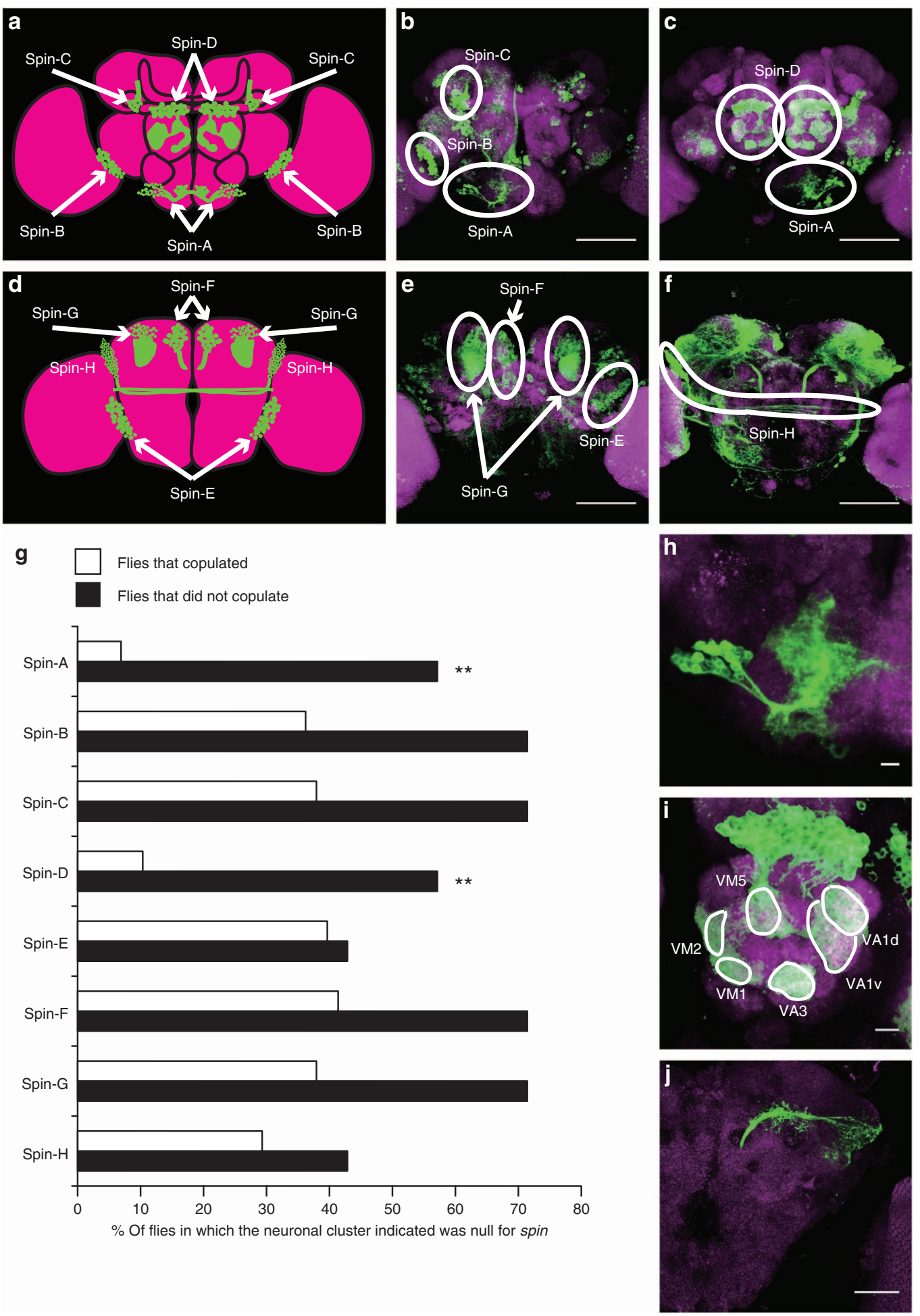

Figure 2 | Two neuronal clusters reduced receptivity when homozygous for spin in the otherwise spin heterozygous brain. (a-f) Eight neural clusters repeatedly labelled as spin mutant cells were present in non-copulating mosaic females. Four clusters (spin-A to -D) in the anterior brain shown as a schematic (a) or reconstructed images $(\mathbf{b}, \mathbf{c})$. Another four clusters (spin-E to $-\mathrm{H}$ ) in the posterior brain shown as a schematic (d) or reconstructed images (e,f). Scale bar, $100 \mu \mathrm{m}$. (g) The proportions of flies in which the indicated clusters were spin homozygous in the two fly groups, that is, copulating flies (open bars) and non-copulating flies (filled bars). Female flies that did not copulate within a 1-h observation period were classified as 'non-copulating.' By examining 902 mosaic females, we recovered seven non-copulating females that were then used to estimate the spin-homozygous ratio for each neuron cluster. To determine the neuronal spin-homozygous ratio for copulating flies, the brains from 58 flies were scored. These 58 flies were arbitrarily chosen from fly pools that yielded a non-copulating mosaic fly. Pairwise comparisons of the spin mutant ratio between the copulating and non-copulating groups for each cell cluster revealed statistically significant differences only in the spin-A and -D clusters. The statistical significance of differences was evaluated by the Fisher's exact probability test $\left({ }^{\star \star} P<0.01\right)$. (h) The structure of spin-A cluster neurons labelled as a MARCM clone. (i,j) The structure of spin-D cluster neurons labelled as a MARCM clone, which are shown for somata and dendrites in antennal lobe glomeruli (VA1d, VA1v, VA3, VM1, VM2 and VM5; i) and for axons projecting to the mushroom body and lateral horn (j). Scale bar, $10 \mu \mathrm{m}(\mathbf{h}, \mathbf{i})$ and $50 \mu \mathrm{m}(\mathbf{j})$. 

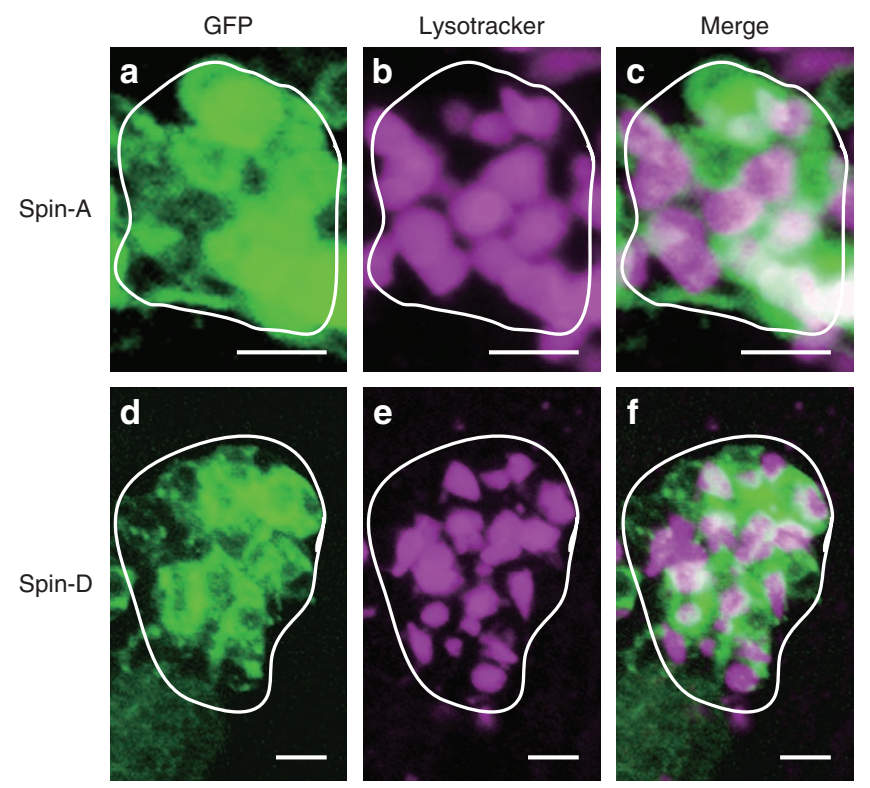

Figure 3 | Spin-A and -D MARCM clones examined for the expression of lysotracker. GFP fluorescence monitoring spin-GAL4 expression (left-hand side panel), lysotracker signals (middle panel) and their merged image (right-hand side panel) are shown for spin-A (a-c) and spin-D (d-f) clusters that are spin mutant clones generated by MARCM. Scale bar, $5 \mu \mathrm{m}$.

Table 1 | Genotype combinations of spin-A to spin-H clusters in seven mosaic flies that did not copulate.

Individual flies that did not copulate

No. 20 No. 267 No. 326 No. 463 No. 589 No. 607 No. 635

\section{Spin-A}

Spin-B

Spin-C

Spin-D

Spin-E

Spin-F

Spin-G

Spin-H

$\begin{array}{cccc}+ & + & + & \\ + & + & ++ & \\ + & + & ++ & + \\ + & & ++ & + \\ + & & + & \\ & + & + & + \\ & & ++ & + \\ & & + & +\end{array}$

\begin{tabular}{|c|c|c|}
\hline \multicolumn{3}{|l|}{+} \\
\hline & + & + \\
\hline & + & \\
\hline+ & & \\
\hline & + & + \\
\hline+ & + & + \\
\hline
\end{tabular}

' Indicates that the cluster was unilaterally positive for GFP staining and thus $\operatorname{spin}^{p 2}$ homozygous.

++ ' Indicates that the cluster was bilaterally positive in mosaic females (the fly identification number is shown in the top raw) that did not copulate.

Spin-A to - $\mathrm{H}$ clusters were chosen for analysis because these were the type of clusters that were repeatedly labelled (thus mutant for spin) in seven non-copulating mosaic females (labelled in at least three such females).

Note that, in fly no. 635, neither the spin-A nor the spin-D cluster was mutant for spin, even Note that, in fly no. 635 , neither the spin-A nor the spin-D cluster was mutant for spin, even
though the fly did not copulate, presumably reflecting a contribution of yet unidentified neurons to the receptivity. to the spin mutant females, in which no copulation was observed. This is presumably because the neural pathway involving spin-A cluster neurons remained functional even after the spin-D pathway was impaired by the spin knockdown in Or47b neurons, whereas in spin mutant females both the spin-A and -D pathways were disrupted.

Mechanistic target of rapamycin signalling is required for normal sexual receptivity. spin knockdown in Or47b neurons is much more effective in reducing sexual receptivity than neural activity block in the same group of neurons (Fig. 5b versus Fig. 6b). This implies that Spin signalling in the neurons is pivotal for regulating sexual receptivity.

As Spin has been shown to activate mechanistic target of rapamycin (mTOR) in starvation-induced autophagy ${ }^{19,20}$, we examined the possible effect of mTor inactivation on spindependent sexual receptivity. When spin or mTor was singly knocked down, females exhibited only a moderate reduction in receptivity (Fig. 6f). In contrast, when both spin and mTor were knocked down simultaneously, the females showed a striking reduction in receptivity (Fig. 6f). This synergistic effect of spin and $m$ Tor supports the hypothesis that these two proteins cooperate to regulate female sexual receptivity.

\section{Discussion}

In this study, we identified two groups of neurons whose malfunction resulted in a significant decline in female sexual receptivity: a population of olfactory projection neurons with its dendritic field in the VA1v glomerulus of the antennal lobe (spin-D) and a cluster of local neurons in the suboesophageal ganglion (spin-A). It is of interest to note that the location of projection neuron somata appears to coincide with SP11, to which Tompkins and $\mathrm{Hall}^{21}$ assigned the role of controlling female sexual receptivity by classical mosaic analysis.

VA1v is one of the three fru-expressing sexually dimorphic glomeruli $^{15,16}$ and receives synapses from Or47b neurons that are known to respond to a conspecific odour common to both sexes $^{14}$. It is therefore likely that the Or47b-VAlv sensory pathway participates in the species recognition rather than in the discrimination of the sex within the species. A recent study showed that wild-type males, but not Or47b mutant males, vigorously court a hydrocarbon-deficient target male and this courtship is suppressed when the target male has been perfumed with 7-tricosene (7-T) (ref. 22), a male-predominant pheromone, which is also present in females at a low level. This observation suggests the intriguing possibility that Or47b neurons accelerate mating in both females and males by responding to compounds
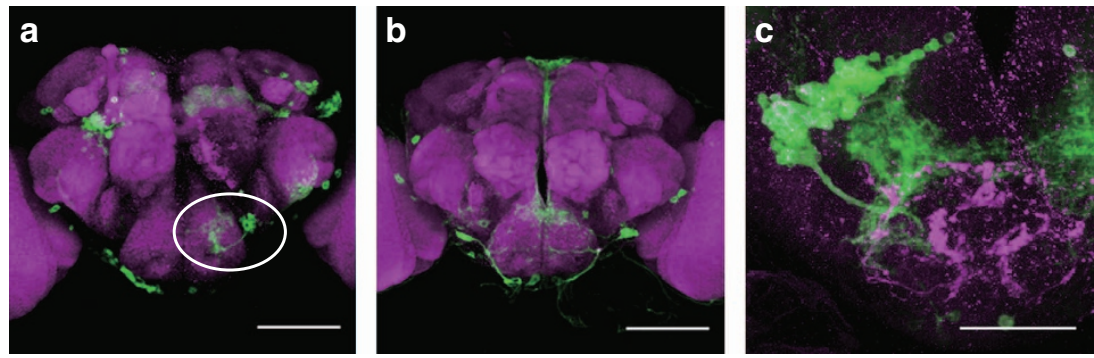

Figure 4 | Anatomical and neurochemical characteristics of spin-A cluster neurons. (a) The spin-A cluster was labelled with mCD8::GFP (circled). (b) The spin-A cluster was never labelled when the fly carried Chat-GAL80, which inhibits the GAL4 function in cholinergic neurons. Anterior view. Scale bar, $100 \mu \mathrm{m}$. (c) Spin-A cluster neurons have arborizations interdigitating with ppk-positive axon terminals. The ppk-mCherry fusion gene was used to label ppk neurons in the brain that carried a spin-A MARCM clone marked with mCD8::GFP. Scale bar, $50 \mu \mathrm{m}$. Green: spin-GAL4-positive cells (a-c). Magenta: neuropil staining with nc82 $(\mathbf{a}, \mathbf{b})$ or mCherry expression $(\mathbf{c})$. 

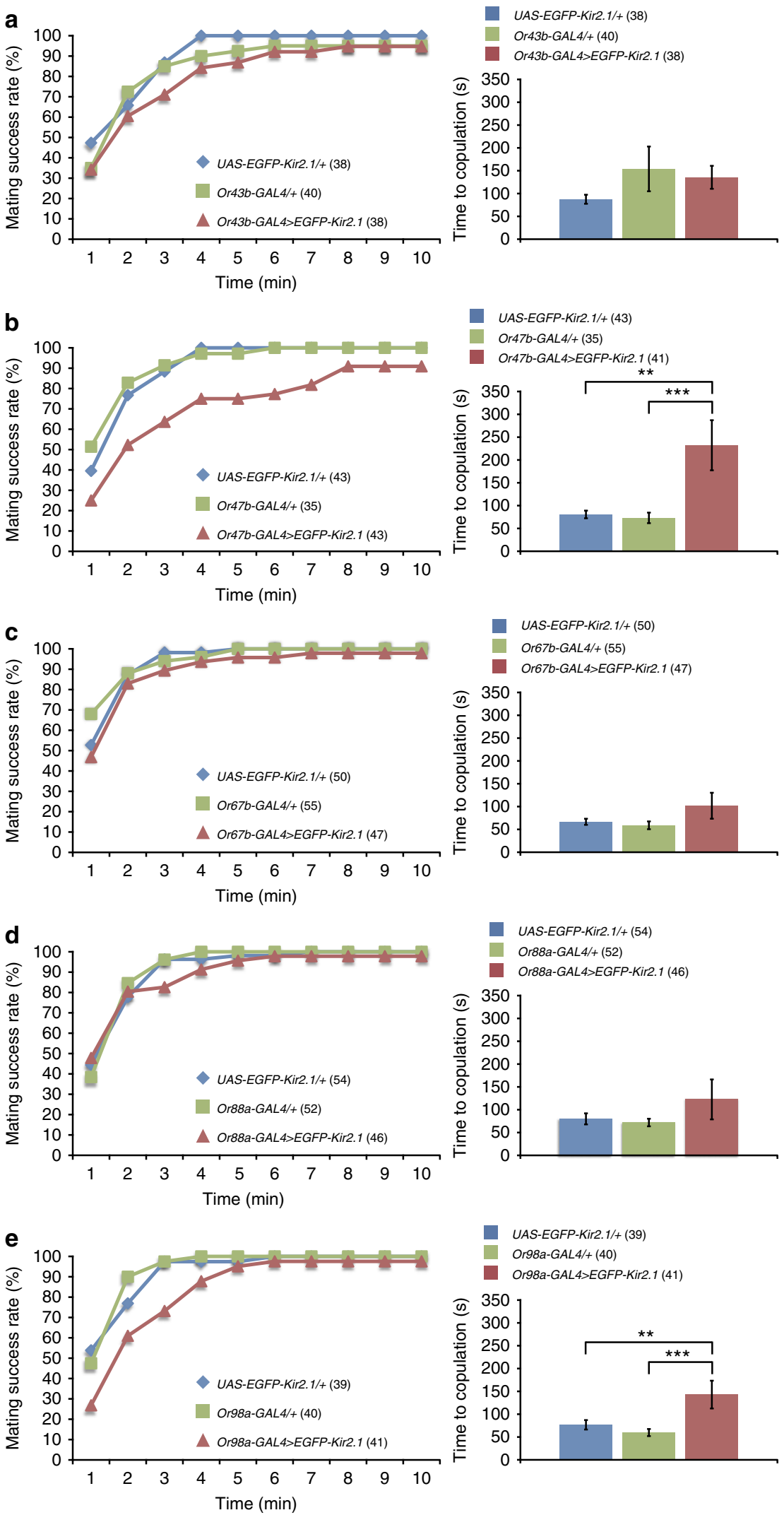

Figure 5 | Differential effects of blocking olfactory receptor neuronal activity on sexual receptivity. The cumulative number of pairs copulating over time (relative to the total pairs observed: left-hand side graphs) and the time to copulation (mean \pm s.e.m: right-hand side graph) were compared among the females in which olfactory receptor neurons expressing either Or43b (a), Or47b (b), Or67b (c), Or88a (d) or or98a (e) were prevented from firing. The statistical significance of differences was evaluated by the Kruskal-Wallis analysis of variance followed by Scheffe's $F$ test for the measure of time to copulation $\left({ }^{\star \star \star} P<0.001 ;{ }^{\star \star} P<0.01\right)$. The number of flies examined is shown in parentheses. 

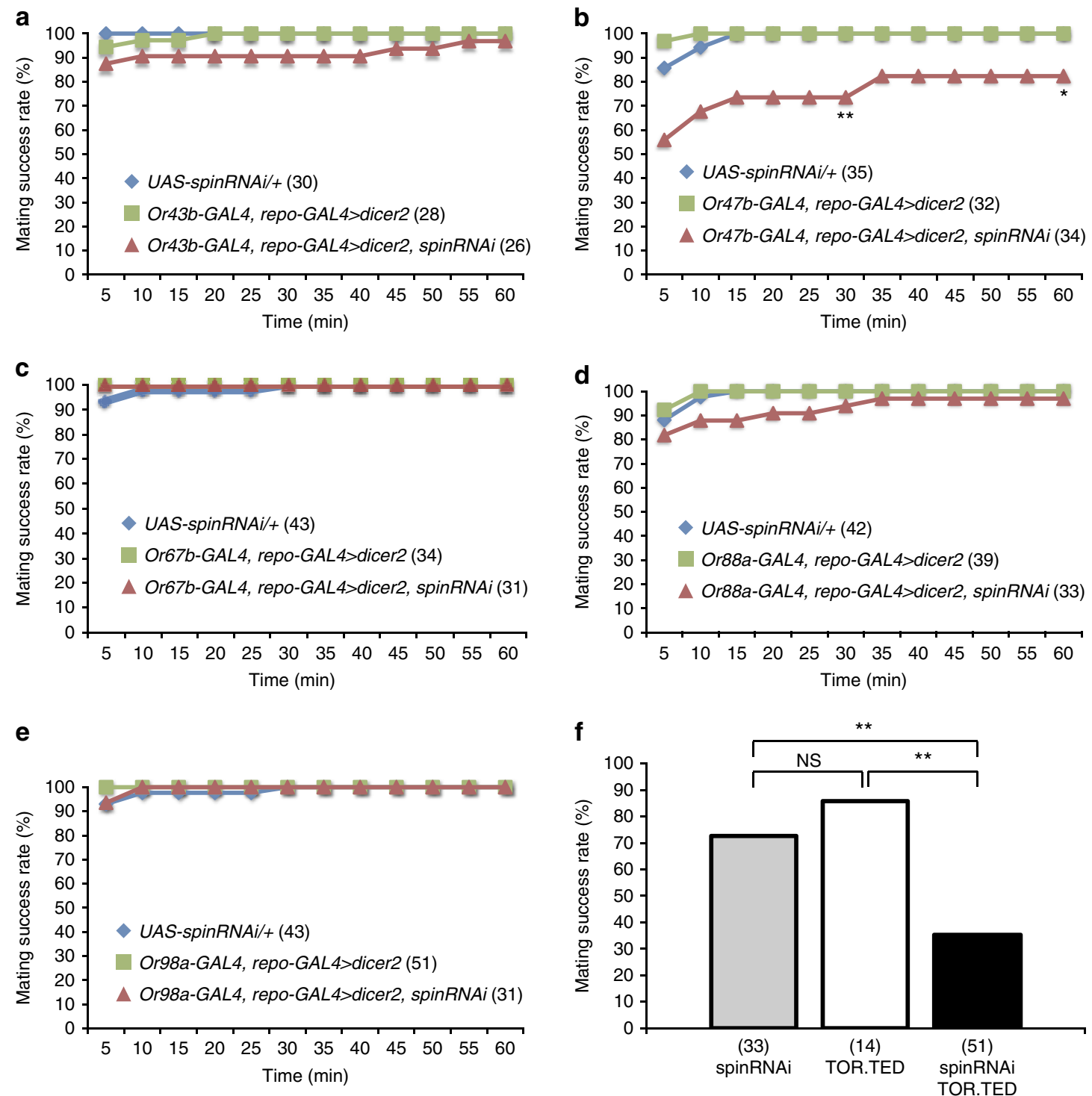

Figure 6 | The effect of spin RNAi expressed in olfactory receptor neurons and of mTOR inhibition on sexual receptivity. (a-e) The cumulative number of pairs copulating over time (relative to the total pairs observed) was compared among the females in which spin RNAi was expressed in olfactory receptor neurons expressing either $\operatorname{Or} 43 b(\mathbf{a}), \operatorname{Or} 47 b(\mathbf{b}), \operatorname{Or} 67 b(\mathbf{c}), \operatorname{Or} 88 \mathrm{a}(\mathbf{d})$ or Or98a (e). Note that repo-GAL4 and UAS-dicer2 were used to enhance the effect of spin RNAi on sexual receptivity. The statistical significance of differences was evaluated by the Fisher's exact probability test with Bonferroni correction for the cumulative copulation success $\left({ }^{\star \star} P<0.01 ;{ }^{\star} P<0.05\right)$. (f) Synergy of $m$ Tor and spin in the regulation of female receptivity. Although the mating success rate in a 1-h observation period was only moderately reduced by the elav-GAL4-mediated expression of either dominant-negative $m$ Tor (TOR.TED, $85.7 \% ; n=14$ ) or spin RNAi (spinRNAi; $72.7 \% ; n=33$ ), simultaneous expression of these two (TOR.TED + spinRNAi) resulted in a marked decrease in the receptivity $(35.3 \% ; n=51)$. The statistical differences were evaluated by the Fisher's exact probability test with Bonferroni correction, or by the $\chi^{2}$-test for the comparison in which the expectation frequency exceeded 5 in all parameters ( ${ }^{\star \star} P<0.01$; not significant (NS) $P>0.05$ ). The number of flies examined is shown in parentheses.

common to both sexes, and that the excitatory state produced by Or47b neuron activities is modulated negatively by $7-\mathrm{T}$ in males and positively by $7-\mathrm{T}$ in females. In fact, 7 - $\mathrm{T}$ has been shown to enhance female sexual receptivity, and amputation of the female antennae abrogates this effect of 7-T (ref. 23). On the other hand, Or47b neurons do not respond to a male-specific pheromone, cis-vaccenyl acetate ${ }^{14}$, which reportedly accelerates female acceptance of a courting male ${ }^{24}$. Or47b neurons make synapses in the VA1v glomerulus on the projection neurons extending axons to a region in the $l h$ pheromone responsive field that is sexually dimorphic ${ }^{10}$. In this region, many fru-expressing neurons intersect and some of the command outputs to control courtship are likely initiated there, at least in males $7,25,26$.
On the other hand, the local interneurons composing the spin-A cluster have not been described previously, and thus nothing is known about their input-output relationships. The suboesophageal ganglion is well-known as the centre for gustation, which has a central role in pheromone perception in mating ${ }^{27}$. Recent studies have suggested that, upon copulation, the $p p k$ and fru double-positive uterine mechanosensory neurons projecting to the ventral ganglia trigger the conversion of female physiology and behaviour from the virgin type to the mated type $^{28,29}$. In this study, we found that spin-A cluster neurons potentially receive inputs from $p p k$-positive neurons of a nonuterus origin (Fig. 4c and Supplementary movie 1), whose roles in female receptivity need to be addressed. In addition, a few 
$d s x$-expressing interneurons in the ventral ganglia with an ascending axon that terminates in the suboesophageal ganglion have been implicated in the control of female sexual receptivity ${ }^{30}$. Therefore, it is envisaged that the spin-A cluster neurons integrate multiple sensory inputs for switching the female physiology and behaviour upon copulation and their outputs are relayed by the ascending interneurons to the brain. Spin-A cluster neurons express neither fru nor $d s x$, and, therefore, are unlikely to be sexually dimorphic. It is tempting to speculate that the spin-A cluster originally engaged in metabolic homoeostasis later became a key regulator of sexual receptivity, specifically in females, where this cluster had become integrated into a femalespecific circuitry that controls mating behaviour.

We found that the reduction in female sexual receptivity was much more pronounced when spin was knocked down than when neuronal activity was suppressed in Or47b neurons (Figs $5 \mathrm{~b}$ and $6 \mathrm{~b})$. It might be that spin knockdown impaired neural development, leading to a more severe phenotype than that induced by the acute inhibition of neural activities. Note, however, that no apparent structural deficits were detected in spin-homozygous neurons that had developed as a MARCM clone in the otherwise heterozygous background, even though they exhibited high levels of lysotracker signals, indicative of lysosomal malfunction (Fig. 3). Although spin homozygous flies underwent progressive neurodegeneration ${ }^{4,8}$, mosaic flies carrying spin mutant cells did not suffer from a similar symptom, presumably because nearby wild-type (spin heterozygous) cells complemented, at least in part, functional deficits in spin mutant cells. We therefore consider that the behavioural phenotype observed in these mosaic flies is not an indirect consequence of global neurodegeneration. An alternative possibility is that not only 'ionotropic' (electrical activity) but also 'metabotropic' (molecular signalling) functions are important for these neurons to attain a high level of female sexual receptivity, and the Spin protein is the critical component in the latter process. Indeed, Spin is an important signalling molecule that mediates cell death ${ }^{4,8}$ and lysosome reformation associated with mTOR activation following starvation ${ }^{19,20}$. Spin is also suggested to be an efflux permease that mediates release of lysosomal digests into cytosol $^{19}$. The mTOR signalling pathway composes the core system for the homoeostasis of nutritional states ${ }^{31,32}$. Mating induces drastic changes in nutritional demands in females as they begin massive production of eggs to oviposit after copulation. In fact, females develop a preference for protein-rich diets after mating, and this change in food preference appears to be mediated by mTOR signalling ${ }^{32}$, which is, in part, stimulated by the neural action of the sex peptide transferred from a male during copulation ${ }^{31,33}$. An attractive hypothesis is that the food preference and sexual receptivity in females are both regulated by mTOR signalling, in which Spin is involved.

Our study has identified two neuronal groups critically involved in the regulation of sexual receptivity, and this opens the way to elucidation of the sophisticated decision-making circuitry for adaptive changes in female behaviour at the singlecell level.

\section{Methods}

Flies. Flies were raised on a cornmeal-agar-yeast medium at $25^{\circ} \mathrm{C}$. The variants we used are described in the FlyBase database (http://flybase.org/). Wild-type Canton-S flies were used as the control. $\operatorname{spin}^{P 1}$ and $\operatorname{spin}^{P 2}$ are strains carrying a $P$-element insertion at the spin locus, and were described in Nakano et al ${ }^{4}$. All RNAi strains were purchased from the Vienna Drosophila RNAi Center. elav ${ }^{c 155}$, an enhancer-trap GAL4 driver, was used to induce UAS-spin ${ }^{+}$type I,

UAS-spinRNAi or UAS-TOR.TED expression in all neurons and repo-GAL4 in most of glia. To enhance the knockdown effect, UAS-dicer-2 was introduced into the flies. Chat-GAL80 (ref. 34) was used to inhibit the GAL4 function in cholinergic neurons.
Behavioural assays. The animals were raised at either 25 or $29^{\circ} \mathrm{C}$ by the time of mating behaviour assays, which were conducted at $25^{\circ} \mathrm{C}$. To observe mating behaviour, virgin females were collected at eclosion and placed individually in food vials for 4-7 days. Each female fly was transferred to a round mating chamber ( $8 \mathrm{~mm}$ in diameter; $3 \mathrm{~mm}$ in height) with a wild-type virgin male. The behaviour of the fly pair was recorded using a video recorder. To estimate the level of female sexual receptivity, the cumulative number of copulating pairs in a 1-h observation period was counted and the average time to copulation was compared among the fly groups subjected to different types of manipulation or different genotypes.

MARCM analysis of female sexual behaviour. Somatic clones were produced using the MARCM method as described previously ${ }^{5}$. The flies used for MARCM analysis were obtained by crossing females of $y$ hs-flp; FRTG13 tub-Gal80 and males of $w$; FRTG13 UAS-mCD8::GFP spin ${ }^{P 2} / C y O$; spin-GAL4. Embryos were collected within $24 \mathrm{~h}$ of egg laying and heat-shocked at $37^{\circ} \mathrm{C}$ for $180 \mathrm{~min}$. The mating behaviour assays for the mosaic flies were essentially identical to those described above, except that the single females were tested twice with a different male to minimize the inclusion of false-negative events in which the female fortuitously failed to copulate (irrespective of the spin genotype) in the first test.

Histology. Fixation and immunohistochemical staining were carried out as described previously ${ }^{35}$ using the following antibodies and dilutions: rabbit polyclonal anti-GFP (1:1,000; Molecular Probes, Eugene, OR), rat monoclonal antiElav (1:100; Developmental Studies Hybridoma Bank, Iowa city, IA), mouse monoclonal anti-Repo (1:50; Developmental Studies Hybridoma Bank), mouse monoclonal nc82 (1:20; Developmental Studies Hybridoma Bank), Alexa488conjugated goat anti-rabbit IgG (1:200; Invitrogen, Carlsbad, CA) and Alexa546conjugated goat anti-mouse IgG (1:200; Invitrogen). To label lysosomes, brains were stained with $100 \mathrm{nM}$ Lysotracker Red DND-99 (Molecular Probes) for $30 \mathrm{~min}$ without fixation. Stacks of optical sections at 1 or $2 \mu \mathrm{m}$ were obtained with an LSM 510 META confocal microscope (Carl Zeiss, Oberkochen, Germany) and were processed with ImageJ software (ver. $1.40 \mathrm{~g}$ ).

\section{References}

1. Sander van Doorn, G., Edelaar, P. \& Weissing, F. J. On the origin of species by natural and sexual selection. Science 326, 1704-1707 (2009).

2. Manning, A. The control of sexual receptivity in female. Drosophila Anim. Behav. 15, 239-250 (1967).

3. Suzuki, K., Juni, N. \& Yamamoto, D. Enhanced mate refusal in female Drosophila induced by a mutation in the spinster locus. Appl. Entomol. Zool. 32 235-243 (1997).

4. Nakano, Y. et al. Mutations in the novel membrane protein Spinster interfere with programmed cell death and cause neural degeneration in Drosophila melanogaster. Mol. Cell Biol. 21, 3775-3788 (2001).

5. Lee, T. \& Luo, L. Mosaic analysis with a repressible cell marker for studies of gene function in neuronal morphogenesis. Neuron 22, 451-461 (1999).

6. Kimura, K., Hachiya, T., Koganezawa, M., Tazawa, T. \& Yamamoto, D. Fruitless and Doublesex coordinate to generate male-specific neurons that can initiate courtship. Neuron 59, 759-769 (2008).

7. Kohatsu, S., Koganezawa, M. \& Yamamoto, D. Female contact activates malespecific interneurons that trigger stereotypic courtship behavior in Drosophila. Neuron 69, 498-508 (2011).

8. Dermaut, B. et al. Aberrant lysosomal carbohydrate storage accompanies endocytic defects and neurodegeneration in Drosophila benchwarmer. J. Cell Biol. 170, 127-139 (2005).

9. Sweeney, S. T. \& Davis, G. W. Unrestricted synaptic growth in spinster-a late endosomal protein implicated in TGF- $\beta$-mediated synaptic growth regulation. Neuron 36, 403-416 (2002).

10. Jefferis, G. S. X. E. et al. Comprehensive maps of Drosophila higher olfactory centers: spatially segregated fruit and pheromone representation. Cell 128, 1187-1203 (2007).

11. Couto, A., Alenius, M. \& Dickson, B. J. Molecular, anatomical, and functional organization of the Drosophila olfactory system. Curr. Biol. 15, 1535-1547 (2005).

12. Fishilevich, E. \& Vosshall, L. B. Genetic and functional subdivision of the Drosophila antennal lobe. Curr. Biol. 15, 1548-1553 (2005).

13. Baines, R. A., Uhler, J. P., Thompson, A., Sweeney, S. T. \& Bate, M. Altered electrical properties in Drosophila neurons developing without synaptic transmission. J. Neurosci. 21, 1523-1531 (2001).

14. van der Goes van Naters, W. \& Carlson, J. R. Receptors and neurons for fly odors in Drosophila. Curr. Biol. 17, 606-612 (2007).

15. Kondoh, Y., Kaneshiro, K. Y., Kimura, K. \& Yamamoto, D. Evolution of sexual dimorphism in the olfactory brain of Hawaiian Drosophila. Proc. R. Soc. Lond. B 270, 1005-1013 (2003).

16. Stockinger, P., Kvitsiani, D., Rotkopf, S., Tirián, L. \& Dickson, B. J. Neural circuitry that governs Drosophila male courtship behavior. Cell 121, 795-807 (2005). 
17. Dickson, B. J. Wired for sex: the neurobiology of Drosophila mating decisions. Science 322, 904-909 (2008).

18. Yamamoto, D. Brain sex differences and function of the fruitless gene in Drosophila. J. Neurogenet. 22, 309-332 (2008).

19. Rong, Y. et al. Spinster is required for autophagic lysosome reformation and mTOR reactivation following starvation. Proc. Natl Acad. Sci. USA 108, 7826-7831 (2011)

20. Yu, L. et al. Termination of autophagy and reformation of lysosomes regulated by mTOR. Nature 465, 942-946 (2010).

21. Tompkins, L. \& Hall, J. C. Identification of brain sites controlling female receptivity in mosaics of Drosophila melanogaster. Genetics 103, 179-195 (1983).

22. Wang, L. et al. Hierarchical chemosensory regulation of male-male social interactions in Drosophila. Nat. Neurosci. 14, 757-762 (2011).

23. Grillet, M., Dartevelle, L. \& Ferveur, J. -F. A Drosophila male pheromone affects female sexual receptivity. Proc. R. Soc. B 273, 315-323 (2006).

24. Kurtovic, A., Widmer, A. \& Dickson, B. J. A single class of olfactory neurons mediates behavioural responses to a Drosophila sex pheromone. Nature 446, 542-546 (2007).

25. Ruta, V. et al. A dimorphic pheromone circuit in Drosophila from sensory input to descending output. Nature 468, 686-690 (2010).

26. von Philipsborn, A. C. et al. Neuronal control of Drosophila courtship song. Neuron 69, 509-522 (2011).

27. Koganezawa, M., Haba, D., Matsuo, T. \& Yamamoto, D. The shaping of male courtship posture by lateralized gustatory inputs to male-specific interneurons. Curr. Biol. 20, 1-8 (2010).

28. Yang, C. -H. et al. Control of the postmating behavioral switch in Drosophila females by internal sensory neurons. Neuron 61, 519-526 (2009).

29. Häsemeyer, M., Yapici, N., Heberlein, U. \& Dickson, B. J. Sensory neurons in the Drosophila genital tract regulate female reproductive behavior. Neuron 61, 511-518 (2009).

30. Rezával, C. et al. Neural circuitry underlying Drosophila female post-mating behavioural responses. Curr. Biol. 22, 1155-1165 (2012).

31. Ribeiro, C. \& Dickson, B. J. Sex peptide receptor and neuronal TOR/S6K signaling modulate nutrient balancing in Drosophila. Curr. Biol. 20, 1000-1005 (2010).

32. Vargas, M. A., Luo, N., Yamaguchi, A. \& Kapahi, P. A role for S6 kinase and serotonin in postmating dietary switch and balance of nutrients in $D$. melanogaster. Curr. Biol. 20, 1006-1011 (2010).

33. Carvalho, G. B., Kapahi, P., Anderson, D. J. \& Benzer, S. Allocrine modulation of feeding behavior by the sex peptide of Drosophila. Curr. Biol. 16, 692-696 (2006).
34. Kitamoto, T. Conditional disruption of synaptic transmission induces male-male courtship behavior in Drosophila. Proc. Natl Acad. Sci. USA 99, 13232-13237 (2002).

35. Kimura, K., Ote, M., Tazawa, T. \& Yamamoto, D. Fruitless specifies sexually dimorphic neural circuitry in the Drosophila brain. Nature 438, 229-233 (2005).

\section{Acknowledgements}

We thank T. Sakai for Chat-GAL80; the Bloomington Stock Center, Kyoto Genetic Resource Center and Vienna Drosophila RNAi Center for numerous stocks; the Developmental Studies Hybridoma Bank for antibodies; and S. Abe for secretarial assistance. This work was supported in part by Grants-in-Aid for Scientific Research (24113502, 23220007, 1802012 to D.Y., and 24570082 and 23115702 to M.K.) from the Japanese Government Ministry of Education, Culture, Sports, Science and Technology (MEXT); a grant from the Strategic Japanese-French Cooperative Program from the Japan Science and Technology Agency (JSPS) to D.Y.; a Life Science Grant from the Takeda Science Foundation to D.Y.; and a Grant-in-Aid for JSPS fellows (no. 22-6772) to A.S.

\section{Author contributions}

A.S., M.K. and D.Y. designed the research, A.S. performed most of the experiments, M.K. performed the experiment shown in Fig. 6f, K.Y. and K.E. produced ppk-mCherry transgenic fly strain, and A.S. and D.Y. wrote the paper.

\section{Additional information}

Supplementary Information accompanies this paper at http://www.nature.com/ naturecommunications

Competing financial interests: The authors declare no competing financial interests.

Reprints and permission information is available online at http://npg.nature.com/ reprintsandpermissions/

How to cite this article: Sakurai, A. et al. Select interneuron clusters determine female sexual receptivity in Drosophila. Nat. Commun. 4:1825 doi: 10.1038/ncomms2837 (2013).

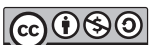

This work is licensed under a Creative Commons AttributionNonCommercial-ShareAlike 3.0 Unported License. To view a copy of this license, visit http://creativecommons.org/licenses/by-nc-sa/3.0/ 